\title{
Granular Space and the Problem of Large Numbers
}

\author{
V. I. Konushko \\ Protvino, Moscow, Russia \\ E-mail:konushko@mail.ru \\ Received February 21, 2010; revised February 28, 2011; accepted March 2, 2011
}

\begin{abstract}
Two and a half thousand years ago the ancient atomists made a suggestion that space has a cellular structure, is material and consists of elementary cells. In 1900 Plank found the elementary length $\mathrm{L}^{*}=10^{-33} \mathrm{~cm}$. This notion has been widely used in modern physics ever since. The properties of granular space are studied in this article on the assumption that a three-dimensional material cell with the size of Planck's elementary length is the only material for the construction of the whole Universe. This approach allows one to account for such mysterious phenomena as inertia, ultimate velocity of transfer of material body interactions and huge difference between gravitational and Coulomb forces - the so called "Large Numbers Problem", as well essence of electric charge and Pauli exclusions principle.
\end{abstract}

Keywords: Problem of Large Numbers

\section{Introduction}

The concept of space is one of the most important conceptions forming the system of our knowledge.

Is the space infinite or not? Is it just a relation between material bodies or does it exist independently? Is the space a container for matter observable even in the absence of material bodies? Is the space uniform from one point to another or are there some selected directions? Is it neutral or does it direct bodies inside it? Do we know its properties intuitively without any external influence on our brain or do we acquire these properties from experience? These are the questions made in different times with respect to a phenomenon named space.

The conception of discreteness is as old as that of continuity. It goes back to ancient atomists and can be regarded as one of the first solutions of Zeno's of Elea aporias. However, it should be noted that in spite of successful application of the idea of discreteness to describe the structure of matter, the operating conception for the space and time structure was nevertheless that of continuity.

A big step in solving the problem of space structure was made in 1900 by Plank [1]. In that year Plank's constant $\mathrm{h}$ was born. Plank researched the irradiation of black bodies. He was attracted by the universality of this irradiation, which turned out to be independent of the size as well as of the shape of the irradiating body or of the properties of the vessel walls. While the reasons of this universality were searched for, the problem of the standards of length, mass and time appeared. These standards were to be established from the principles not appealing to any substance including elementary particles. They were only to be expressed through the fundamental constants, i.e. the speed of light $\mathrm{c}$, Newton gravitational constant $\mathrm{G}$ and the quantum of action $\mathrm{h}$ found from the irradiation law. Only these three constants Plank could take as fundamental ones. From those three constants only one value with the dimension of the length $L^{*}$, fundamental mass $m^{*}$ and time quantum $\tau^{*}$ could be constructed:

$$
\begin{aligned}
& L^{*}=\sqrt{\hbar G / c^{3}} \approx 1.6 \times 10^{-33} \mathrm{~cm}, \\
& m^{*} \approx 10^{-5} \mathrm{~g}, \\
& \tau^{*}=L^{*} / c \approx 10^{-43} \mathrm{sec} .
\end{aligned}
$$

An elementary clot of matter with the mass $m^{*}$ got the name of a plankeon. Three values $L^{*}, m^{*}$ and $\tau^{*}$ play a major role in the theory of elementary particles as well as in the Big bang model. However, physical meaning of the elementary length $L^{*}$ has still not become clear.

There is a wide-spread opinion that the Plank's length could bring light to numerous mysteries of micro- and macro-worlds. "Only one value has in the existing theory a clear and available interpretation - that is the Plank's length. Whence still, if not from here, is it possible to start researches of particles? It is quite possible that only 
the physics of the $10^{-33} \mathrm{~cm}$ region will help us to understand the physics of elementary particles", - wrote Wheeler [2]. Okun' also saw the significance of this value [3]. "It seems more and more probable that physics on a Plank's scale determines not only all the physics of low energies but the whole picture of the Universe as well", - he remarked.

The problems of the micro world make us consider the picture of the universe where the idea of discreteness must play the role not less than that of continuity. It is necessary that the discreteness should participate in the description of quantum objects quite naturally without being artificially brought in the context of continuum notions.

A serious step in comprehension of the space structure was made by Beckenstein [4]. Considering the thermodynamics of black holes he supposed that the entropy of a black hole was proportional to its square: $\mathrm{S} \sim \mathrm{A}$. But the square $A$ has the dimension of the length squared and there appeared a problem how to make the expression for the entropy dimensionless. A hypothesis was put forward that the entropy of a black hole must have the following form:

$$
S \approx \frac{\alpha \times A}{\left(L^{*}\right)^{2}},
$$

where the coefficient $\alpha$ must be calculated basing on some other ideas. This coefficient was later on calculated by Hawking [4]. It turned out to be $1 / 4$. The value $\left(L^{*}\right)^{2}$ is the minimal square of an elementary object. Whereas Plank found the minimum value of the elemen- tary length, Beckenstein and Hawking indirectly intro- duced the minimum size of the elementary square. Using the above-mentioned, we only have to take the next step towards the generalization up to a minimum three-dimensional object in order to establish the space structure.

We suppose that the meaning of the Plank length $L^{*}$ lies in the fact that physical space has a cellular structure and consists of material three-dimensional cells with the size of $L^{*} \sim 10^{-33} \mathrm{~cm}$. Thus, we suppose that eternal, invariable, primary matter-protomatter - exists in the form of an elementary cell of the size of fundamental length $L^{*}$.

According to Wheeler [2], "it is cells of this size that make up space on its deepest level". All our observations and experiments have been and are performed in a material Universe. It is quite unreasonable to expect that a theory developed under such conditions will be applicable in an empty Universe.

As we have already mentioned, no matter what the nature of original mater is, if cannot produce either a point, or an infinite thin line or an ideal plane. The only and the simplest object which can be created by the material Universe is a bubble. When coming in contact with one another these bubbles turn into polyhedrons, i.e. threedimensional geometries or cells.

There are five types of polyhedrons which, when arranged in parallel, can cover a three-dimensional space so that they would be franked to one another by their whole faces (Figure 1):

The most economical geometry is a fourteen-sided polyhedron: the volume being the same, it takes the least material to make its face.

The entropy of the black hole acquires quite a transparent physical meaning - it is equal to the number of elementary cells forming the surface of this object:

$$
S \approx \frac{\alpha \times A}{\left(L^{*}\right)^{2}}=N .
$$

Therefore, a mysterious and amazing quantity - entropy - appears to be connected with the structure of space. Further on, this fact will help us to see the reason of irreversibility of physical processes though all conservation laws are convertible in time. "The arrow of time" will be considered in more detail in our subsequent papers.

\section{The Size of Elementary Particles}

Before considering the above-mentioned problem let us raise one of the "native" questions which are most difficult to answer. Doesn't the assumption, that the Universe consists of only one element, inappropriately simplify the reality? To answer it we should keep in mind that the



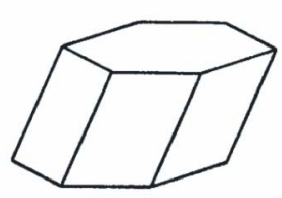

2

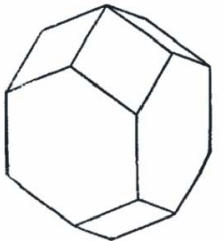

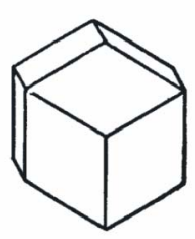

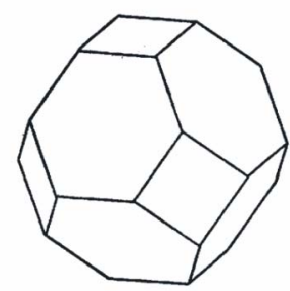

Figure 1. Three-dimensional polyhedrons. (1. parallelepipeds; 2 . prisms with centrally-symmetric six-sided bases; 3. twelve-sides polyhedrons; 4 . twelve-sides polyhedrons; 5 . fourteen-sides polyhedrons.) 
world constants give us a notion of the size of an elementary particle - Comption's wavelength. For protons, it is

$$
\lambda_{p}=\frac{\hbar}{m_{p} \times c} \approx 2.1 \times 10^{-14} \mathrm{~cm} .
$$

Experiments give a somewhat larger value of proton size: $r \approx 7 \times 10^{-14} \mathrm{~cm}$.

Simple calculations give us the number of elementary cells of which this particle consists, $\sim 10^{60}$. The number of nucleons in the whole Universe is $\sim 10^{80}$. It is rather amazing, but in the number of structural elements any elementary particle is hardly inferior to the Universe.

The creation of an elementary particle requires a supplementary quantity of matter of mass. Since the whole space is filled with cells, the rest mass when it forms a particle shell, must deform both internal and external cells. The internal cells form the body of the particle which literally "occupies" or confines these space cells. The deformation of external cells makes the essence of physical fields. Radial deformation creates electrostatic and gravitational fields. Tangential (torsion) deformation results in magnetic, gravitational - magnetic and vortex electrostatic fields, as well as a particle spin.

Matter means the substance which is used to form elementary cells. Particle mass is the amount of matter used to form this corpuscle.

In creating a particle the outer cells have to be slightly pushed apart thus forming an excess of matter in the surrounding space. This excess is exactly equal to the mass of the particle itself and, according to the Einstein formula for excessive energy, $E=m c^{2}$. It is just this excess of matter that provides the basis for introducing the concept of potential energy: it becomes quite clear from where the Space gets excessive energy as a particle moves in different physical fields.

Kinetic energy means the amount of matter carried by a particle; this matter moves ahead of the particle carrying it forward and makes the motion wavy by nature. Moreover, it is this matter that forms new particles as two corpuscles collide. In our article "Weak Interaction and the nature of virtual of particles" we have discussed more comprehensively the motion of photons and particles.

Nowadays we haven't the slightest idea of what the electric charge and the spin mean. The reason lies in our erroneous view of leptons as point particles. Erroneous is the interpretation of experiments on lepton - lepton scattering. In this case the matrix element does not contain any form-factors of these particles which would take into account their complex structure. The absence of such a structure is closely connected with the lepton size. Now let us consider, as a counterexample, the process of bil- liard balls elastic scattering which is considered as a collision of material points though their structure is much more complex than that of particles.

According to the present-day concepts, the size of an electron $r_{e}<10^{-17} \mathrm{~cm}$, and this comes into conflict when the density of matter inside an electron and a proton is under consideration. The electron mass is 2000 times less than the mass of a proton, and the density of electron matter in this case is $10^{9}$ times as much as that proton. All this leads to an absurd chain: the less the mass of an elementary particle, the smaller its size and the more the density of matter inside it.

The rule - the more the mass of a particle, the smaller its size - is supported by an experiment performed on a relativistic heavy ion collider (RHIC). The mass of a particle $J / \psi$ is more than three times larger than that of a proton and, as it follows from the experiment, its size is three times smaller.

The size of a particle is its most important characteristic which must be determined only by the world constants $\hbar, c, G$ and by the mass of a particle $m$. Three quantities pretend to be the radius of electron: the Compton wavelength $\lambda$, the classical radius $r_{0}$ and gravitational radius $r_{g}$ :

$$
\begin{aligned}
& \lambda=\frac{\hbar}{m c} \approx 3.86 \times 10^{-11} \mathrm{~cm}, \\
& r_{0}=\frac{\hbar}{m c} \frac{e^{2}}{\hbar c}=\frac{\hbar}{m c} \times \alpha \approx 2.8 \times 10^{-13} \mathrm{~cm}, \\
& r_{g}=\frac{G \times m}{c^{2}} \approx 10^{-55} \mathrm{~cm} .
\end{aligned}
$$

Any theory having a claim on a correct description of the microcosm must be able to calculate the fine structure constant $\alpha$, which acts as the electromagnetic interaction constant, and in the radius $r_{0}$ it serves just as a scale factor. Since the electric charge is the same for all elementary particles, the value of $e^{2}$ cannot determine the size of numerous elementary corpuscles which differ greatly in mass. The gravitational radius of an electron is much smaller than that of an elementary cell, and therefore is not discussed here. It inevitably follows that the size of any structure - free particle is only dictated by its Compton wavelength. The structure of a particle, however, increases its size just slightly, like in case of a proton. Over fifty years ago both Yukawa and Landau proposed that the size of an electron is equal to its Compton length.

On arriving at this decision we finally can understand such notions as the mass, the electric charge and the spin of elementary particles. These problems will be studied in detail in our article "Weak Interaction and the Nature of Virtual Particles" where we calculated both the mass of one elementary cell $m_{\text {cell }}$ and its density $p_{\text {cell }}$ and found 
a unique relation between an elementary cell and a Wboson. An elementary cell is a generalized image of an object which nature gives us though its world constants.

In imaginary experiments used widely by Galileo, Newton and Einstein we can see with our own eyes all these mysterious natural phenomena thus doing away with the tragedy of blindness. Being prisoners of "pointness" we would never solve these fundamental problems. Besides, there are infinities which have been poisoning the life of theorists for about a hundred years provided that particles are considered to be points.

Let us go back to the proton. Its large mass creates surface tension strong enough to produce inside a particle clusters of deformed internal cells. Such formation has already acquired the name of quarks. Quark "confinement" becomes now quite transparent, and the similarity of quarks and leptons can be accounted for by the fact that inside either of them there are no clusters of deformed cells.

$\alpha$ proton consists of $N_{p}$ deformed cells:

$$
N_{p}=\left(\frac{\hbar}{m_{p} c}\right) \times \frac{1}{\left(L^{*}\right)^{3}} \approx 10^{57} \text {. }
$$

The deformation of such a huge number of cells is so queer that it gives grounds to introduce into theory such objects as gluons and a sea of virtual quart-antiquark pairs despite the fact that all these objects have a material basis, i.e. they consist of material cells.

Even in collisions of an electron with another particle its internal cells are just elastically deformed without creating any new internal formations, and this point is considered nowadays as electron "pointness".

\section{Particle Motion. Ultimate Velocity. Enigma of Inertia}

To observe the motion of a particle we must make an imaginary experiment by increasing the elementary cell up to the size of a small cube. The particle begins to move only when there is a difference in cell deformation behind and in front of the particle, i.e. a deformation gradient. The amount of matter required for it and surrounding the particle in asymmetric way is called kinetic energy and the cell deformation gradient is referred to as acceleration.

Since cells process elastic properties, the motion of this additional matter has a wave nature creating some kind of "a centaur": a wave-particle predicted by the Broglie. An electron is only carried by the wave never becoming part of it. After colliding with another particle the electron loses the prefix "wave". The process of transmitting either a part of matter (kinetic energy) or the whole matter has an exchange character. In collision the matter carried by the shell-particle having reached the target-particle having reached the target-particle finds itself between a hammer and an anvil.

The enormous quantity of deformed cells participating in the collision leads us to introducing into our theory the notion of a "virtual" exchange particle. In this immense "pot" a strong deformation of space cells makes up all kinds of cluster providing the right to introduce such notions as a sea of quark-antiquark pairs, current quarks and gluons. That is why there is such a strong difference between the masses of current and constituent quarks. This real collision process enables us to understand this "spin disaster" as well.

The introduction of structural functions into the matrix element is the first raw attempt of describing a complex collision act where up to $10^{80}$ deformed space cells take part. Such a huge amount of deformed cells participating in a collision act is responsible for the fact that all the events in the Universe are not local and our mathematical description of an event will always be just approximate. The nonlocal character of elementary particles and interactions gives rise to a false concept about the violation of the principle of casuality and the principle of equation invariance under Lorentz's transformation.

But both a line, a point and plane are started mathematical concept, and in real space such objects do not exist at all. Any event in the Universe occupies a certain space-time area, and the fact that we attribute the coordinates $X, Y, Z$ and $t$ to this event just says that this event has really happened, that is, something has occurred in the Universe, something has changed in a certain region of space in a definite time. In all our equations we mentally reduce this region to a point and the use of formfactors is just a weak attempt to account for a colossal complexity of the collision process. We have so far prepared the ground for discussing the following fact: no one has ever observed the motion of a physical object with respect to other objects with its velocity exceeding at a definite moment of time the speed of light in vacuum $\left(c \approx 3 \times 10^{10} \mathrm{~cm} / \mathrm{s}\right)$ excluding the giant "scissors" effect.

In three-dimensional cell space all motion is conducted by cells themselves and the speed of light is the parameter of their elastic properties. In other words, for the cells there exists a maximum deformation that defines the maximum possible velocity. The deformed cells cannot achieve the velocity of deformation transfer above the maximum. Consequently no particle can exceed this velocity. The history of tachyons repeat that of flogiston and caloric. The planned experiment on the discovery of neutrino velocity exceeding the speed of light is in our opinion doomed to failure.

The phenomenon of inertia has been known for many 
centuries and Galilei, Huygens, Descartes and Newton polished the wording of this mysterious phenomenon. Any macroscopic body moves through liquid or gaseous media as one whole pushing the molecules of these materials. However, in order to break the bonds between the water molecules a body, e.g. a submarine, must spend a part of its kinetic energy (in other words, matter) that it is carrying to compensate for the binding energy between these molecules. Along with this, the momentum of the submarine is diminished, which leads to the decrease of the deformation gradient of the cells surrounding the submarine. Finally the body stops.

An elementary particle, which itself consists of cells, is moving in the cell space also pushing and deforming the other cells in front of it without spending any kinetic energy, since in free space the notion of the binding energy between the adjacent cells is absent.

If a liquid or a gas were not viscous at all, a body moving in these media would not meet any resistance ("Eiler's paradox"). It is in this way that an elementary cell moves through the cells of field-free space. The space viscosity is equal to " 0 "!

For the last five years the experiments with the use of a relativistic heavy ion collider (RHIC) have allowed us to reproduce at a microscopic scale quark-gluon plasma formed by collisions of gold nuclei flying almost at the speed of light.

Some physicists are surprised to see that a quark-gluon media is practically free of viscosity and so presents the most ideal liquid among all the known liquids [8]. It is rather difficult to get rid of the idea that this ideal liquid accounts for the absence of viscosity in space.

But there is a fundamental difference between the wave motion of elementary particles and photons and a sound wave or a wave in a liquid.

The propagation of sound in the air is the motion of elastic deformations caused in the air rather than the motion of air masses, for example, the wind as a photon or a particle moves, space carries a mass which is the mass of a virtual object, i.e. the corner-stone of quantum theory.

Even this peculiarity alone of the space body motion established a crushing psychological barrier under the necessity to allot the invisible and imperceptible object, i.e. the space, a real material structure. Mysterious character of space makes some physicists go back to the notion of ether, others - to create a new generalized image of the space-vacuum, endowing both notions with fantastic properties.

The basis of the inertia principle appears to be the absence of the absorption of matter connected with a moving object, which changes the deformations of cells situated at each time moment near the given body. However, after the body leaves this region, the space cells obtain their previous form if there are no other bodies or fields there. Even sweeping all the stars out of the Universe, nevertheless the space and the inertia will still exist. Thus, the cell space contradicts the Mach's principle.

\section{Enigma of Large Numbers}

Any theory is only worthy of notice when it contains numbers.

For further investigation of the properties of the elementary cell we revert to two fundamental laws, i.e. Newton's gravitation law and the Coulomb's law:

$$
F_{N}=G \cdot \frac{m_{1} \cdot m_{2}}{R^{2}}, F_{K}=K \cdot \frac{q_{1} \cdot q_{2}}{R^{2}} .
$$

Here physical mechanisms veiled by false simplicity of their mathematical expression are of interest. The laws are very similar in form. Noticeable is a similar dependence both on distance and charges. But the most interesting is the relative value of these forces. From the previous experience it is known what key role the dimensionless values like the Reynolds's number, Knudsen's number, Mach's number, etc. play in understanding physical phenomena.

Let us find the ratio of these forces for two electrons:

$$
\frac{F_{K}}{F_{N}}=\frac{K \times q_{e}^{2}}{G \times m_{e}^{2}} \approx 4.17 \times 10^{42} .
$$

The value is amazing and there hardly exists a physiccist who has never thought what it means. Many prominent scientists attempted to get this number [4]. Most known is the Dirac's attempt. He divided the age of the Universe by the time during which a light beam passes the distance equal to Compton's length of a proton:

$$
\frac{T u}{t_{p}} \approx \frac{10^{18}}{10^{-24}} \approx 10^{42} .
$$

Feynman [7] joked that this number could be obtained by dividing the Earth volume by the volume of an aphid, but what have they to do with this number? In fact the situation becomes much more complicated if to recollect that even nowadays the number of elementary particles is about one thousand and for each particle there is its infinitely large number and, besides, the number of particles is growing with disastrous speed.

All these infinite numbers must be obtained from one and the same assumption. Perfect will be a solution when theory will operate only with fundamental constants $G, c$, $h$.

To make the solution more clear we shall do an operation, the meaning of which will further on become evident. Let us consider an interaction where the constant is $1 / \alpha \approx 137$ times bigger that the electromagnetic con- 
stant ( $\alpha$ is the fine structure constant):

$$
\frac{g^{2}}{\hbar c}=1 \text {. }
$$

Now let us find the ratio of this interaction force to Newton's force. Then for $e, \mu$ and $p$, respectively, we obtain:

$$
\begin{aligned}
& \frac{F_{g}}{F_{N} e}=\frac{g^{2}}{G \times m_{e}^{2}}=\frac{\hbar \times c}{G \times m_{e}^{2}} \approx 10^{44}, \\
& \frac{F_{g}}{F_{N} \mu}=\frac{g^{2}}{G m_{\mu}^{2}}=\frac{\hbar \times c}{G m_{\mu}^{2}} \approx 10^{40}, \\
& \frac{F_{g}}{F_{N} p}=\frac{g^{2}}{G m_{p}^{2}}=\frac{\hbar \times c}{G m_{p}^{2}} \approx 10^{38} .
\end{aligned}
$$

We do not give numbers for other particles and only note that this ratio is rapidly decreasing with the increase of the particle mass. Intriguing is the result for the heaviest clot (if it exists), i.e. plankeon:

$$
\frac{F_{g}}{F_{N} m^{*}}=\frac{g^{2}}{G m^{* 2}}=\frac{\hbar c \times G}{G \times \hbar c}=1 \text {. }
$$

Now let us recollect that matter (particle mass) forms only a steady stable surface shell, but a "particle body" consists of space cells. Or else, the mass of a particle deforms both internal and external cells as if "cutting" from space a mini-volume, which later on we call an elementary particle. Therefore, only the cells of the surface layer participate in the interaction.

Let us find the square of one elementary cell using the ideas of Beckenstein and Hawking:

$$
S_{c} \approx\left(L^{*}\right)^{2}=\frac{\hbar G}{c^{3}} \approx 10^{-66} \mathrm{~cm}^{2} .
$$

Now let us recollect that the size of the particle, as it was previously found, is equal:

$$
r=\text { const } \times \frac{\hbar}{m c},
$$

and the coefficient const can only be of an order of a unit (for a proton const is equal to 3 ).

Let us estimate the number of cells for one layer $10^{-33} \mathrm{~cm}$ thick, which makes the surface of a particle:

$$
N=\frac{S}{S_{c}}=\frac{\hbar^{2} \times c^{3}}{m^{2} \times c^{2} \times \hbar \times G}=\frac{\hbar \times c}{G \times m^{2}} .
$$

The number of cells on the particle surface is equal to:

$$
\begin{aligned}
& N e=\frac{S e}{S c}=\frac{\hbar c}{G m_{e}^{2}} \approx 10^{44}, N \mu=\frac{S \mu}{S c}=\frac{\hbar c}{G m_{\mu}^{2}} \approx 10^{40}, \\
& N p=\frac{S p}{S c}=\frac{\hbar c}{G m_{p}^{2}} \approx 10^{38}, N m^{*}=\frac{S m^{*}}{S c}=1 .
\end{aligned}
$$

for $e, \mu, p$ and $m^{*}$, respectively.

Comparing the ratio of the interaction forces (9) with the number of cells making the particle surface (14), we obtain an overwhelming result: these numbers are equal identically!

What is then the physical meaning of this amazing equality? It appears to be rather transparent. Let us consider the simplest case when particles are pressed to each other and their surfaces touch. Then in our contrived strong interaction the matter of all cells, making the nearest to the surface layer, participate; and in the gravitational interaction there is only one cell which participates!

Turning back to the Coulomb's force, we obtain the equality:

$$
\left(\frac{F_{K}}{F_{N}}\right)_{\text {cell }}=\frac{\alpha \times N}{1}
$$

which is a key to the solution of the mystery of large numbers.

Now the physical meaning of the fine structure constant $\alpha$ becomes clear: in the Coulomb interaction of two charged particles at a distance equal to the size of the particle only a part of cells of the surface layer equal to $\alpha \approx 1 / 137$ of their total number takes part. Besides, the cells form layers around the particle and in each layer the deformation of cells is identical, since the cells of one layer are at the same distance from the particle. As the difference in force in these interactions lies only in the number of participating cells, then the dependence of these two fundamental forces on the distance becomes similar because the forces are saturated with matter from one and the same layer. Moreover, an elementary cell will "allow" us later on to prove that the potential of both gravitational and Coulomb's interactions is inversely proportional to the distance:

$$
\varphi \sim \frac{1}{r}
$$

and, consequently, the force:

$$
F \sim \frac{1}{r^{2}} .
$$

Physical meaning of other interactions also becomes clear: the more cells from the layer participate in the interaction, the stronger is the interaction. However, the constant of any interaction cannot be more than a unit:

$$
\frac{g^{2}}{\hbar c} \leq 1
$$

since at $g^{2}=\hbar c$ all cells of the layer take part in the interaction, which, in its turn, imposes a limit on the binding energy of two particles: 


$$
E c \leq 1 / 2 m c^{2},
$$

where $m$ is the mass of a lighter particle of the pair.

A vigorous Coulomb interaction brings us to the assumption that there must be a profound reason for such a large number of cells to participate in the interaction. We shall call this phenomenon the effect of elementary cells collectivization.

\section{The Essence of Electric Charge}

Milliken and other scientists have shown in their experiments that electric charges consist naturally of discrete constant portions. A positron has the same amount of electricity as an electron. A more striking example is that all other charged particles have charges exactly equal in value, for instance, the positive charge of electron.

According to the present-day views, there is a difference between an electron and proton, probably, the biggest for elementary particles. But their charges are equal to a high degree of accuracy. It is not clear yet what doesn't permit an electron to decay as well as what determines the exact value of its charge.

The existence of an electric charge in two forms is, of course, its fundamental property. The conservation law and relativistic invariance are also its mysterious features. In an isolated system the full electric charge, i.e. the algebraic sum of positive and negative charges, remains constant. If we measuring a charge in different reference system, we get the same number which drastically differs from measuring the total mass carried by a moving particle: the higher velocity, the larger the total mass, $m=m_{0} \times \sqrt{1-v^{2} / c^{2}}$.

It is difficult to give up the idea that if we had an accelerator with $E \approx 10^{19} \mathrm{GeV}$ and discovered experimentally all the elementary particles which space can create we would not answer any of the above questions. Thus, we have to study the real structure of space already today.

A colossal difference between the Coulomb force and gravitation can only be explained by geometries. Let us consider the simplest analogy. Cut a convex lens of a whole piece of glass. Make a convex line on the glass with one move of cutter. Then, after breaking the glass one can see that the second part has a concave surface, and the radii of curvature of these surfaces are absolutely the same.

When creating electric charges Nature acts in a similar wave, in an imaginary experiment, if we increase an electron to the size of a football we can see that the surface of this particle is made up of clusters of deformed cells resembling segments of the football design. And, if we are not mistaken with the signs, these clusters are concave for the electron (negative curvature) and convex for the positron (positive curvature). The question arises: how many clusters are sited on the electron surface? We can answer this question by considering the constant fine structure $a$ :

$$
\alpha=\frac{e^{2}}{\hbar c}=\frac{1}{137.0359} .
$$

This unattractive number has been agitating the minds of physicists for nearly a hundred years. Feynman said: “... this is one of the greatest accursed mysteries of physics - a magic number we have got and don't understand at all. It might be said that this number has been written by 'God's hand' but we don't know what moved his pencil".

If the only construction for the Universe is a threedimensional elementary cell, the Universe "knows" only natural numbers. E. Cronecker, a mathematician, was rather sagacious when he said that the Lord had created natural numbers, and all the rest was man's handiwork meaning that the role of the Load was played by Nature. If this is the case, the quantity $\alpha$ should be an echo of a whole number. Since the shape of an elementary particle differs a little from being spherical, one can suggest that the number $\pi$ is involved here. Then,

$$
1 / \alpha \times 4 \pi=1722 \text {. }
$$

The number 1722 is accurate enough but it should be taken into account that $\alpha$ has been varying rather widely for the last hundred years, beginning with 136. The number 1722 has a simple physical meaning: it means that the surface of any charged particle consists of 1722 clusters, whereas only the cells of one cluster take part in Coulomb interaction. Hence it follows that all charged particles have equal electric charges even though their masses differ greatly. This fact verifies again an absolute dependence of the particle size on its mass. This dependence is vividly reflected by the Compton length $\hbar$ :

$$
r=\lambda=\frac{\hbar}{m c} .
$$

In our articles we paid attention time and again to the false concept of an electron as a point particle with $r_{e}<$ $10^{-17} \mathrm{~cm}$. There are not any point objects in the world and they cannot exist. Pointness means the absence of a structure, i.e. indivisibility into components. The number $10^{-17} \mathrm{~cm}$ only means that if there are structural formations inside an electron, they are less than $10^{-17} \mathrm{~cm}$, and this figure has nothing to do with the size of the electron itself.

Although a more massive particle has a smaller surface, its cluster contains fewer cells, and a big mass of one cell compensates for the shortage of cells materially.

The electron surface consists of $N$ cells: 


$$
N=\frac{4 \pi r_{e}^{2}}{\left(L^{*}\right)^{2}}=7.3 \times 10^{45},
$$

where $L^{*}=\sqrt{G \hbar / c^{3}}$ - is the Planck length or the particle size, and every cluster contains $4.17 \times 10^{42}$ cells. The quantity of cells would take part in the Coulomb interaction of an electron and positron if they would be brought into contact. In this case the convex surface of one cluster (positron) fully coincides with the concave surface of an electron cluster. In gravitational interaction, however, only one cell would participate in this case! So we are able to unravel the secrets of extreme weakness of gravitational interaction between elementary particles as well as the mystery of big numbers the solution of which Dirac was looking for the Universe.

Our studies into the granular space structure enabled us to find all these infinitely big numbers in the structure of elementary particles without turning to the Universe.

It should be noted that a positron in a real experiment cannot be at the distance $r_{e}=3.86 \times 10^{-11} \mathrm{~cm}$ when it is in contact with electron because the number of cluster cells is $4.17 \times 10^{42}$ and the positron surface consists of $\sim 10^{45}$ cells. The positron in this case simply cannot be placed in "the bed of Procrusten" of electron cluster. The deformed space cells with inevitably push aside the positron at the distance a determined again by the constant $\alpha$ :

$$
\alpha=\frac{\hbar}{m_{e} c} \cdot \frac{\hbar c}{e^{2}}=0.529 \times 10^{-8} \mathrm{~cm},
$$

where $2 \alpha$ is the positron size, $\alpha$ is the hydrogen atom size.

\section{Nature of Energy Levels}

It is of high interest to observe in an imaginary experiment the structure of deformed cells as they gradually move away from the particle. The structure of the cells near a planceon is the simplest and the most prominent since it only consist of one cell.

The mass of this particle is:

$$
m^{*}=\sqrt{\hbar c / G} \approx 2 \times 10^{-5} \mathrm{~g}
$$

and its size

$$
L^{*}=\sqrt{\hbar c / G^{3}} \approx 1.6 \times 10^{-33} \mathrm{~cm},
$$

It should be stressed again that $L^{*}$ is nothing but the Compton length of the planceon which demonstrates a rigid relation between the particle mass and size mentioned repeatedly in [6].

Moving away from this particle we can observe a wonderful phenomenon - a gradual decrease in space cell deformation gives rise to cluster of collectivized space cells.

This may occur in a layer lying at the distance $r_{2}=4 L^{*}$ because this nearest layer can be densely covered with cluster consisting of four cells. The next cluster has nine cells in a layer spaced at the distance $r_{3}=9 L^{*}$ from the planceon. In the most natural way we can detect energy levels at the distance $r_{n}$ from the planceon:

$$
r_{n}=\frac{\hbar}{m^{*} c} \times n^{2}
$$

For the first time energy levels were referred to by $N$. Bohr in 1913. It is easily seen that the number of clusters on the first energy level is two, on the second - eight, on the third - eighteen, and so on. As we have already noted, this is due to the fact that clusters must closely fill their relevant layer of cells. Every cluster is characterized by their specific features in the structure embodied in the quantum numbers: $n, l$ and $m$, thus revealing the mystery of Pauli's exclusion principle. On the first energy level space forms only two clusters, and that is why only two electrons can be sited on it, irrespective of the nuclear charge. On the second level - eight electrons; this is the exact number of clusters formed by space on this level, and so on.

The Pauli exclusion principle plays a pivotal role in our understanding of countless physical and chemical phenomena, ranging from the periodic table of elements to the dynamics if white dwarfs and neutron stars. It has defied all attempts to produce a simple and intuitive proof, despite being spectacularity confirmed by number and accuracy of it.

Wolfgang Pauli remarked in his Nobel Prize lecture (13 December 1946): “Already in my original paper I stressed the circumstance that I was unable to give a logical reason for the exclusion principle or to deduce it from more general assumption. I had the feeling, and I still have it today, that this is a deficiency. The impression that the shadow of some incompleteness fell here on the bright light of success of the new quantum mechanics seems to me unavoidable".

The intrigue connected with Pauli's principle is that the second level can have no more than eight electrons not because these particles "avoid" each other if they have the same quantum numbers but because on the second energy level space allots only eight "seats" to the electrons and the quantum numbers $n, l$ and $m$ reflect a complex structure of clusters which consist of a huge number of deformed elementary cells.

A characteristic feature of science is that we must be able to describe phenomena so that we could say something intelligible without exhaustive data and hand. It is worth nothing that every new theory asks more new questions than answers the old ones. 
The interaction constant of two planceons $\alpha_{p l}$ is:

$$
\alpha_{p l}=\frac{G\left(m^{*}\right)^{2}}{\hbar c}=1 .
$$

Consequently, with $E_{p l}=m^{*} c^{2} \approx 10^{19} \mathrm{GeV}$, all the interactions become united and the energy levels can be called both gravitational, Colombian and nuclear. All the forgoing enables us to draw the following conclusions.

1) All energy levels are characterized by the formation of clusters (collectivization) of deformed space cells at a definite distance from a particle.

2) Any body generates gravitational energy levels around itself.

The formation of energy levels near a charged particle is similar to the process which occurs in the case of the planceon, with the only difference that the role of one elementary cell (planceon) is played by the whole cluster containing $N=4.17 \times 10^{42}$ cells in the case of electron. Also, a correction should be introduced for a smaller value of the electromagnetic interaction constant $\alpha$. Hence, electron energy levels are placed at the distance $r_{n}$ from the proton in a hydrogen atom:

$$
r_{n}=\frac{\hbar}{m_{e} c} \times \frac{\hbar c}{e^{2}} \times n^{2} .
$$

Of interest is that the ordinary proportionality to squared distance can be found in both Colombian and gravitational interactions, and this regularity has a deep meaning. Feynman writes [7]:"... Nobody has so far managed to represent gravitation and electricity as two different manifestations of one and the same essence".

As it has repeatedly been mentioned, in forming a particle the additional matter, i.e. the rest mass, which tries to be sited in a space closely filled with cells, has to deform both internal and external cells of space. The elementary cells pushed outside make up the reserved matter, which can be transferred by space to other particles, and are referred to as potential energy. The deformed outer cells from the substance called a physical field. It is quite obvious that this mass cannot be larger than the rest mass of a particle being formed - a peculiar law of Archimedes in the microcosm. In its turn, it means that the constant of any interaction cannot exceed unity, $g^{2} / \hbar c \leq 1$.

Many physicists have already realized that the value $g^{2} / \hbar c \approx 15$ is just a phenomenological parameter of a strong interaction at low energies rather than the constant of this interaction. In the article concerned with nuclear forces we can find the constant of nuclear interaction on the basis of the deuton binding energy, $\alpha_{s}=g^{2} / \hbar c$ $\approx 0.09736$.

The same dependence of interactions on distance, Feynman spoke about, has a deep meaning which con- sists in forming clusters of deformed outer cells. A gravitational cluster begins with one elementary cell and then gradually grows as it moves away from the particle. In its turn, the electric cluster of, say, the electron in hydrogen atom begins with an object containing $4.17 \times 10^{42}$ elementary cells and then it grows in the same manner as the gravitational cluster. And only at the distance $a=$ $0.529 \times 10^{-8} \mathrm{~cm}$ the electric clusters area increases by $1 / a$ times and the electron can be placed at last in this bed of Procrustes. The gravitational clusters will increase similarly. Then, as the electron moves far away, the cluster areas of both the interactions grow up forming a stringent sequence, like for planceon, $S_{1}: S_{2}: S_{3} \cdots=1: 4: 9 \cdots$

According to the granular space theory, the electron is not only as a definite distance a from the proton but, besides, it is at rest though it has a huge velocity, a momentum and kinetic energy. At first glance, this statement is paradoxical. The electron velocity in heavy atoms is as high as the velocity of length $c$.

To unravel this paradox we revealed the true physical meaning of velocity in about ten articles considering numerous examples: $\varepsilon=v^{2} / c^{2}$ is the relative value of deformation of elementary space cells. So, the following three quantities: the velocity, momentum and kinetic energy of an electron on the ground energy level of any atom are characteristic not of the motion but the deformation of elementary space cells.

All the physical phenomena considered by us from the standpoint of material discrete space fully confirm Einstein's position "Got doesn't play dice" and save us for ever Bohr's attempt to ascribe indeterminism and uncertainty to space. In more detail it will be discussed in another article.

To our surprise we can observe almost visually the quantization of not only Coulomb fields but also gravitational ones. In experiments we cannot feel gravitational levels of particles because of their small sizes but, as the mass of the object increases, the gravitational clusters become larger, too. In our work "Gravitational levels and the Problem of Microwave Background" we found out that the gravitational levels of the Earth are responsible for the formation of quasi-black-body radiation near the Earth with $T \approx 2.7 \mathrm{~K}$ which is competitive with the "relict" radiation hypothesis.

One of the fundamental properties of electric charge, its existence in two forms, is related, as we have established, to the fact the deformed cells on the surfaces of two particles form clusters consisting of a huge number of cells and having the same concave and convex surfaces for both the particles. In mathematics the properties of curve surfaces were studied by Lobachevsky, Gauss, Rhyman, et al. It is rather amusing that curve surface mathematics enters physics now through the front door 
and not through the back one because we are able to see the curvature of surfaces with our own eyes removing the nonobservability of theory. As we cannot cut only one convex surface of glass without the other part of glass getting a concave surface, so it is impossible to generate a positive charge without a negative one. No doubt, charges can disappear only in pairs, too.

So, we can observe practically visually the fundamental property - the law of charge conservation and its existence in two forms.

In our next articles we are going to consider more comprehensively the mechanisms of both gravitational and Coulomb interactions, and here we want just to note that gravitation can be realized when the deformation of elementary cells beyond two bodies is larger than between them whereas repulsion is characterized by large deformation of cells between the bodies. The difference in cell deformation results in a deformation gradient called force. The curvature of the layers of the deformed cells around a particle or a macroscopic body is a secondary effect.

There is exhaustive experimental evidence that the total charge of a system remains constant as the charge charge carriers move. We have got used to it so that we don't often think about such a wonderful and fundamental fact.

Mass does not possess this property of invariance. The matter carried by kinetic energy forms an object referred to in modern theory as a virtual photon, its structure will be considered in a section concerned with the motion of elementary particles. The kinetic energy of a particle increases as the transferred mass of matter is increased by $1 / \sqrt{1-v^{2} / c^{2}}$ times. The space and a particle carry fast additional substance used to form new particles when a shell particle collides with a target particle. The mass of the electron does not change in this case, its size remains constant, too. As for the moving virtual object (photon), the following rigid ratio is valid: $r=\lambda=\hbar / m c$, where $m=m_{0} / \sqrt{1-v^{2} / c^{2}}$ means the total mass of the electron and the virtual photon.

Let a moving electron has $E_{e}=938 \mathrm{MeV}$ which is equal to the rest energy of proton. In this case the wavelength of the virtual photon (not the electron) $\lambda=2 \times 10^{-14} \mathrm{~cm}$, i.e. it is equal to the size of rest proton. It should be noted that the surface of the virtual photon duplicates that of the electron: it is made up of segments, or clusters of negative curvature, too. Since the mass transferred by it is equal to that of the proton, the cluster will be exactly the same as the cluster on the proton surface, and the decreased area of the cluster is compensated for by a larger mass. To our surprise we have to state that this virtual photon has a charge of proton and, hence, the charge of a moving electron is equal to that of a rest electron thus making the electric charge invariant.

\section{The Birth of an Elementary Particle}

The process of formation of an elementary particle remains as mysterious as the number 1722. But space creates a particle in miserable portions of a second, and if our eyesight were keener, these secrets would have been revealed long ago. Once someone said well that nature laughed at our difficulties.

Now we can set forth just some preliminary considerations having a claim on rigorous proof but, nevertheless, containing a number of important ideas.

It is the shape of the cell, a polyhedron, which evidently plays a leading role in forming a particle. To simplify the problem, let us consult again honeycomb. Every cell is hexahedral in shape, and all the subsequent layers that "dress" are the same hexahedrons. Thus, almost all elementary particles have the same priming surface. Owing to this needle-shaped broken structure, the additional matter - the particle mass - can cover part of space cells deforming them and, with certain conditions met, can form a surface film at least for a short instant, like in the case of resonances. We are, without fail, to reveal these mysterious conditions already today not waiting for the construction of a $10^{19} \mathrm{GeV}$ accelerator, because even this energy will not tell us anything new about the electron structure.

The formation of a particle is a real disaster; the mathematical theory of catastrophes is under rapid development now. Let's consider a simple analogy. The birth of a particle only vaguely resembles the work of clock ratchet-and-pawl. The pawl slides quietly over the ratchet for a while but, when it reaches the end of a notch, it falls and a catastrophe happens.

Similarly, a gradual increase in substance quantity, the mass in this region, causes both the radial and the torsion deformations of cells to increase. The latter defines the spin structure. Charged particles are born in pairs, and this process ands with a catastrophe all of a sudden when the previously independent space cells form clusters of positive and negative curvatures on a closed surface. The process of clusterization and collectivization of elementary cells is the main mystery of elementary particle birth.

As the particle mass increases, the deformation of the inner cells reaches such a point that it is useful for space to create clusters inside the particle, clusters called quarks. The cluster confinement in this case is quite a transparent phenomenon: without external deformation of space cells the quarks simply disappear.

This unusual deformation makes a prerequisite for introducing the conception of color and odor to theory.

Though these processes are still far from being fully 
understood, the birth of a particle does not need either additional fields or mediator-particles. An analogy with a soliton will probably help us to approach the secret of formation of a stable surface of elementary particles. The soliton is assumed to be kept stationary at the cost of a balance between the action of a nonlinear medium and dispersion.

We should add another point to understand the gravitational potential $\varphi$. Let us estimate $\varphi$ on the surface of an electron, a muon and a proton:

$$
\begin{gathered}
\varphi_{e}=G \frac{m_{e}}{r_{e}}=\frac{G \times m_{e}^{2} \times c}{\hbar} \approx 10^{-44} \times c^{2}, \\
\varphi_{\mu}=G \frac{m_{\mu}}{r_{\mu}}=\frac{G \times m_{\mu}^{2} \times c}{\hbar} \approx 10^{-40} \times c^{2}, \\
\varphi_{p}=G \frac{m_{p}}{r_{p}}=\frac{G \times m_{p}^{2} \times c}{\hbar} \approx 10^{-38} \times c^{2} .
\end{gathered}
$$

We have already come across the large numbers $10^{44}$, $10^{40}$ and $10^{38}$ which denote the numbers of cells making up the surfaces of an electron, a muon and a proton. Since the squared velocity $v^{2}$ is a dimension of potential, the value $v^{2} / c^{2}$ shows which part of cells of their total number in a given layer is involved in the interacttion.

Here we want to illustrate with a proton one specific feature of cell behavior: though one cell of proton surface requires substance $10^{8}$ larger than one cell of electron surface, only one cell participates again in the gravitational interaction of two protons at an ultimately close distance.

\section{Conclusion}

In conclusion it should be noted that even the first "steps" of an elementary cell have removed a touch of mysticism and fantasy off quite a number of fundament physical phenomena. The Universe is found to be infinitely simple, as it is only made up of one element, and infinitely complex, as any of its matter clusters consists of an infinite number of elementary cells. So, is space continuous or discrete? It would be safe to say, neither of the two. But this naked negation does not feed our thoughts and thoughts and negatively affects theory. Therefore, the more exact answer is: both.

Space is discrete because it consists of discrete elementary cells, it is also continuous since any deformation of cells in a continuous manner (and not by steps) spreads from one cell to another establishing absolute hundredpercent causality and definiteness our great predecessors Planck, Lorentz, de Broglie, Schrödinger, Dirac and Einstein believed in till the end of their lives.
To solve the problem of large numbers filly explains the smallness of gravitation as compared to other forces acting in the microcosm and does not need a fantastic hypothesis for the existence of extra spatial dimensions. Only a three-dimensional elementary cell enables the Universe to create objects of any complexity. The functional dependence of gravity on distance $f \approx 1 / r^{2}$ must remain constant up to distances $r \approx 10^{-33} \mathrm{~cm}$.

Atom stability is one of the most burning problems of theoretical physics, and any attempt of solving this problem with the use of the Geisenberg inequality is invaliddated by numerous experiments. In our next articles we shall subsequently consider atom stability, Geisenberg inequalities, the foundation of probability consideration of the $\psi$-function and the curvature of four-dimensional space-time in Einstein's theory of gravity, the nature of the microwave background of the Universe, the time "arrow" and other subjects making use of the material structure of granular space and only three world constants.

More and more physicists are aware that space is granular in structure and sets an absolute system of reference [8].

Comparatively a short time ago astronomers discovered a wonderful star picture: groups and clusters of galaxies of various scales form a cellular-netted large-scale structure of the Universe. In our opinion, this large astral cell is born by the cellular structure of the space itself.

Newton shared the opinion that the real space is to some degree an empty box where material bodies move about without affecting the space at all. Einstein's theory of gravity has invalidated this assumption by supporting the view that matter and space are directly interrelated.

The theory of granular space is making another step to the unification of matter and space: any cluster of matter is a complex of fantastically deformed elementary cells of the space itself thus symbolizing the Great Unity of Nature [5].

\section{References}

[1] M. Planck, "The Unity of the Physical Patter of the World," NAUKA, Moscow, 1996, p. 108.

[2] J. Wheeler, "Einsteins Vision," SPRINGER - VERLAG, New York, 1968.

[3] L. Okun', "Introduction to Elementary Particle Physics," NAUKA, Moscow, 1985.

[4] A. Eddington, "The Mathematicl Theory of Relativity," Cambridge University, Cambridge, 1923. P. A. Dirac, "Directions in Physics," Wiley, New York, 1978.

[5] V. Konushko, "Concepts of Granular Space Theory," SPUTNIK, Moscow, 1999.

[6] R. Feynman, "The Feynman Lectures on Physics," Addison-Wesley Publishing Company, London, 1963. 
[7] S. W. Hawking, "The Formation of Particles on Black Holes," Communications in Mathematical Physics, Vol. 43, No. 3, 1975, pp. 199-220. doi:10.1007/BF02345020

[8] T. Jacobson and R. Parentani, "The Echo of the Black
Holes," Scientific American, No. 3, 2006, p. 17. A. Smolin, "Atom's Space and Time," Scientific American, No. 4, 2004, p. 20. 